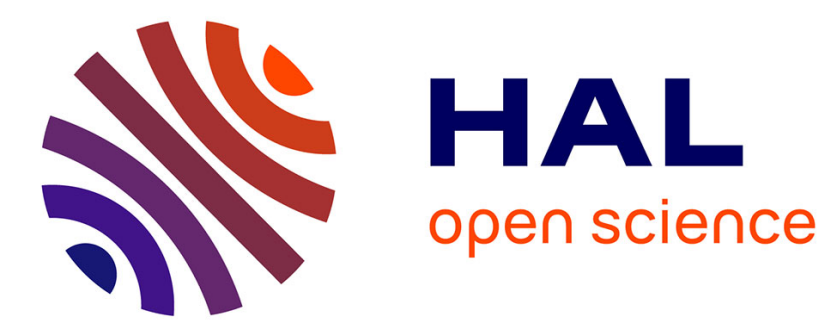

\title{
Ergodic Capacity for Fading Channels in Cognitive Radio Networks
}

\author{
Raouia Masmoudi
}

\section{To cite this version:}

Raouia Masmoudi. Ergodic Capacity for Fading Channels in Cognitive Radio Networks. International Conference on Advanced Technologies for Signal and Image Processing (ATSIP'2018), Mar 2018, Sousse, Tunisia. 10.1109/ATSIP.2018.8364519 . hal-01722855

\section{HAL Id: hal-01722855 \\ https://hal.science/hal-01722855}

Submitted on 5 Mar 2018

HAL is a multi-disciplinary open access archive for the deposit and dissemination of scientific research documents, whether they are published or not. The documents may come from teaching and research institutions in France or abroad, or from public or private research centers.
L'archive ouverte pluridisciplinaire HAL, est destinée au dépôt et à la diffusion de documents scientifiques de niveau recherche, publiés ou non, émanant des établissements d'enseignement et de recherche français ou étrangers, des laboratoires publics ou privés. 


\title{
Ergodic Capacity for Fading Channels in Cognitive Radio Networks
}

\author{
Raouia Masmoudi \\ Gaspard Monge Computer Laboratory (LIGM) \\ University Paris-Est Marne-la-Vallée, France \\ Email: raouia.masmoudi@u-pem.fr
}

\begin{abstract}
In this paper, we consider a Cognitive Radio (CR) system where two type of users try to access to the primary spectrum : a primary user (PU) owning the spectrum license and a secondary user ( $\mathrm{SU}$ ) who does not own the spectrum license. However, the secondary communication is allowed to coexist with the primary communication as long as the interference caused by $\mathrm{SU}$ to $\mathrm{PU}$ is below a tolerable threshold. We study the optimization problem which maximizes the SU's achievable ergodic capacity under different types of power constraints and for different fading channel models. Our goal is to calculate the optimal power allocation strategies for these optimization problems. We show that modelling with Rayleigh fading for the channel between SU transmitter and PU receiver is an advantageous way to ameliorate the $\mathrm{SU}$ ergodic capacity. Furthermore, we consider four combinations of power constraints, since the interference power constraint and the transmit power constraint can be restricted by a peak or an average threshold. We also show that the SU ergodic capacity under average transmit power constraint and average interference power constraint outperforms the one with peak power constraints. In this case, we propose a novel decoupling method. Our method reduces the complexity of the initial problem and makes our initial problem easier to solve.
\end{abstract}

Index Terms-Cognitive radio, fading channels, power allocation, ergodic capacity, interference and transmit power constraints.

\section{INTRODUCTION AND RELATED WORKS}

Recently, in the litterature, a promising and intelligent technology, called Cognitive Radio (CR), have been developed [2], [3] in order to solve the spectrum under-utilization issue [1]. In this context, one can consider two type of users: primary users (PUs) who own the spectrum liscence and secondary users (SUs) who coexist in the primary spectrum with an opportunistic spectrum access manner. These unliscenced users, SUs, are allowed to access to PU's spectrum when it is not used by any PU. However, it is not easy to detect [4] precisely and accurately the PUs vacant bands. Thus, the CR network can allow a simultanoeus SU and PU transmissions provided that SU does not interfere too much with the PU. This SU's transmission strategy is called spectrum sharing [5]-[8].

In the CR context, the power allocation problem should consider the interference caused to the PU by the SU in order

This work is funded by Gaspard-Monge Computer Science Laboratory (LIGM) during my position as a researcher and teaching assistant at University of Paris-Est Marne-la-Valle (UPEM), France. to protect the PU's quality of service (QoS). For fading channels, the study of the power allocation problem in CR networks subject to an average or/and a peak interference power constraint at the PU receiver without considering a transmit power constraint, are made [6], [9]-[11]. In spectrum sharing CR scenarios, the optimal power allocation strategies are studied under both transmit and interference power constraints in [12], [13] and [14]. In [12], the authors focus on an energy-efficient optimal power allocation for fading channels. They studied the ergodic capacity, the outage capacity, and the minimumrate capacity subject to constraints on the average interference power, and the peak/average transmit power constraint. We illustrate that the SU's energy efficiency outperforms under the average transmit power constraint compared to the peak transmit power one. In [14], the authors consider the spectrum sharing context, and study the achievable SU's ergodic capacity and the outage SU's capacity under the peak/average transmit and the peak/average interference power constraints. However, they do not give a rigorous proof for the case where averaged constraints are simultaneously considered. In [19], we study the SU ergodic capacity under both average transmit power constraint and average interference power constraint for different fading channel models. We provide a decoupling method, in order to compute the optimal power allocation policies in our optimization problem. Our method gives an optimal solution and reduces the complexity of the initial problem using Lagrangian tools.

In this paper, we study the SU ergodic capacity, for several fading CR channel models under four various combinations of peak/average transmit and peak/average interference power constraints. This work is an extension to our work in [19]. Our contributions are met: First, we compute the optimal power allocation policy in each optimization problem in order to achieve the maximum SU's capacity. Both, the interference power constraint to protect PU [15]-[17] and the transmit power constraint of SU transmitter are considered. Furthermore, we study the ergodic capacity under several fading CR channel models such that: Rayleigh fading, Log-normal fading and Nakagami fading [18]. Moreover, we consider four combinations of power constraints: peak/average interference power constraint with peak/average transmit power constraint. When the average transmit power constraint and the average interference power constraint are considered, we propose our 
novel decoupling method which reduces the complexity of the initial problem and makes it easier to solve.

The rest of the paper is organized as follows: In section II, we describe the system model and presents several fading channel models. In section III, we consider the different transmit and interference power constraints that are considered in this paper. Then, in section IV, we study the SU's ergodic capacity under different constraints sets combining the peak/average transmit power constraint with the peak/average interference power constraint. We integrate in this section the discussion of some interesting simulation results. Finally, we conclude this paper in section V.

\section{System And Channel Models}

As illustrated in Fig. 1, we consider a CR system model in which a secondary user (SU) is allowed to use the spectrum licensed to a primary user (PU), as the amount of interference power imposed on the PU receiver is within tolerable constraints on the average and peak powers. ${ }^{1}$ We assume that the

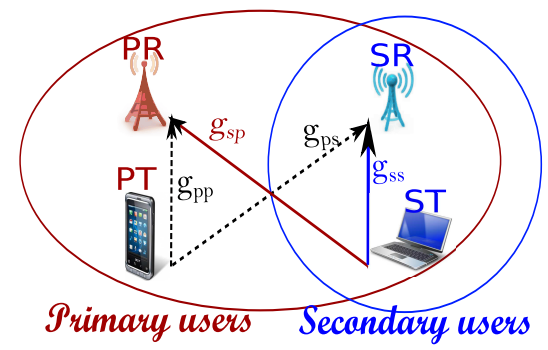

Fig. 1. A spectrum sharing system model in a cognitive radio network.

link between SU transmitter (ST) and PU receiver (PR) is a flat fading channel with the instantaneous channel power gain $g_{s p}[n]$. The SU direct link between ST and SU receiver (SR) is also a flat fading channel characterized by the instantaneous channel power gain $g_{s s}[n]$. At the SR, the received signal $y_{s}[n]$ depends on the SU transmitted signal $x_{s}[n]$ and can be written as follows:

$$
y_{s}[n]=\sqrt{g_{s s}[n]} x_{s}[n]+z_{s}[n],
$$

where $n$ indicates the time index, $z_{s}[n]$ represents the additive white Gaussian noise (AWGN). The noise $z_{s}$ is assumed to be independent random variables with the circularly symmetric complex Gaussian distribution with mean zero and variance $N_{0} .{ }^{2}$ We also assume that the power gains $g_{s s}$ and $g_{s p}$ are independent and identically distributed (i.i.d.) with the probability density function $f\left(g_{s s}\right)$, and $f\left(g_{s p}\right)$, respectively. We assume a perfect channel state informations (CSI) on the power gains $g_{s s}$ and $g_{s p}$ at the ST level. Furthermore, we assume that the interference from PT to SR, denoted here by $g_{p s}$, can be ignored or considered in the AWGN at SR.

In the following, we study three fading channel models [19] such that: Rayleigh fading, Log-Normal fading and Nakagami fading. In each model, we give the expression of the probability density function of the power gain $\left(g_{s s}, g_{s p}\right)$ :

\footnotetext{
${ }^{1}$ We refer by the subscripts $s$ and $p$ to $\mathrm{SU}$, and PU, respectively.

${ }^{2}$ Hereafter, we skip the time index $n$ to simplify the following formulas.
}

- Rayleigh fading $\left(\sigma^{2}\right)$

$$
f_{\text {Rayl }}(x) \triangleq \frac{x}{\sigma^{2}} \exp \left(\frac{-x^{2}}{2 \sigma^{2}}\right), x \geq 0 .
$$

- Log-normal fading $\left(\beta, \sigma^{2}\right)$

$$
f_{\text {LogN }}(x) \triangleq \frac{1}{x \sigma \sqrt{2 \pi}} \exp \left(\frac{-(\ln (x)-\beta)^{2}}{2 \sigma^{2}}\right), x>0 .
$$

- Nakagami fading $(m, \omega, \Gamma)$

$$
f_{N a k}(x) \triangleq \frac{2 m^{m}}{\Gamma(m) \omega^{m}} \exp \left(\frac{-m}{\omega} x^{2}\right), x>0 .
$$

\section{Power Constraints}

From the SU perspective, we can consider either a a peak, or an average transmit power constraint at the ST.

In practice, the peak power limitation is caused by to the non-linearity of the power amplifiers, while the average power is restricted below a certain threshold to maintain a long-term power level.

Let $P\left(g_{s s}, g_{s p}\right)$ represents the instantaneous transmit power at the ST for the channel power gain pair $\left(g_{s s}, g_{s p}\right)$ and $P_{p k}$ represents the peak transmit power threshold, thus, the peak transmit power constraint is given by:

$$
P\left(g_{s s}, g_{s p}\right) \leq P_{p k} .
$$

Also, let $P_{a v}$ denotes the average transmit power threshold, thus, the average transmit power constraint can be represented by the following inequality: ${ }^{3}$

$$
\mathbb{E}\left[P\left(g_{s s}, g_{s p}\right)\right] \leq P_{a v} .
$$

From the PU perspective, we impose either a peak or an average received power constraint at the PR since the SU transmission should not harm the PU's quality of service. We denote by $Q_{p k}$ the peak received interference power at the PR. Then, the peak interference power constraint is given by:

$$
g_{s p} P\left(g_{s s}, g_{s p}\right) \leq Q_{p k} .
$$

And, let $Q_{a v}$ denotes the average received interference threshold at PR, thus, we obtain the following average interference power constraint:

$$
\mathbb{E}\left[g_{s p} P\left(g_{s s}, g_{s p}\right)\right] \leq Q_{a v} .
$$

In the following, by combining these previous power constraints, we obtain the following four constraints sets:

$$
\begin{aligned}
& \mathcal{F}_{1} \triangleq\left\{P\left(g_{s s}, g_{s p}\right):(5),(7)\right\}, \\
& \mathcal{F}_{2} \triangleq\left\{P\left(g_{s s}, g_{s p}\right):(5),(8)\right\}, \\
& \mathcal{F}_{3} \triangleq\left\{P\left(g_{s s}, g_{s p}\right):(6),(7)\right\}, \\
& \mathcal{F}_{4} \triangleq\left\{P\left(g_{s s}, g_{s p}\right):(6),(8)\right\} .
\end{aligned}
$$

\footnotetext{
${ }^{3}$ We denote by $\mathbb{E}[\cdot]=\mathbb{E}_{\left(g_{s s}, g_{s p}\right)}[\cdot]$ the expectation taken over $\left(g_{s s}, g_{s p}\right)$.
} 


\section{The Power Allocation Problems Formulation}

Recently, from a cognitive radio point of view, the SU's channel capacity has attracted a lot of attention. For CR fading channels, ergodic capacity is defined [14] as "the maximum achievable rate averaged over all the fading blocks". Then, we obtain the SU ergodic capacity by solving the following optimization problem:

$$
\begin{aligned}
& C_{e r}=\max _{P\left(g_{s s}, g_{s p}\right) \geq 0} \mathbb{E}\left[\log _{2}\left(1+\frac{g_{s s} P\left(g_{s s}, g_{s p}\right)}{N_{0}}\right)\right] \\
& \text { subject to } \quad P\left(g_{s s}, g_{s p}\right) \in\left\{\mathcal{F}_{1}, \mathcal{F}_{2}, \mathcal{F}_{3}, \mathcal{F}_{4}\right\} \text {. }
\end{aligned}
$$

Hereafter, we will study four problems, where each optimization problem is a maximization of the ergodic capacity defined in (9) under the peak/peak set $\mathcal{F}_{1}$, the peak/average set $\mathcal{F}_{2}$, the average/peak set $\mathcal{F}_{3}$ or the average/average set $\mathcal{F}_{4}$, respectively.

At the optimum, the maximum achievable capacity can be given by:

$$
\begin{gathered}
C_{e r}=\mathbb{E}\left[\log _{2}\left(1+\frac{g_{s s} P^{*}\left(g_{s s}, g_{s p}\right)}{N_{0}}\right)\right]= \\
\int_{g_{s s}} \int_{g_{s p}} \log _{2}\left(1+\frac{g_{s s}}{N_{0}} P^{*}\left(g_{s s}, g_{s p}\right)\right) f\left(g_{s s}\right) f\left(g_{s p}\right) d g_{s s} d g_{s p},
\end{gathered}
$$

where the probability density function over power gains depends on the fading channel model defined in (2),(3) and (4).

To calculate this maximum achievable capacity, we will give either an analytical or a numerical optimal power solution $P^{*}\left(g_{s s}, g_{s p}\right)$.

\section{A. Peak transmit and peak interference power constraints}

In this case, the set of constraints in (9) becomes $\mathcal{F}_{1}$. By combing the two constraints in $\mathcal{F}_{1}$, we can compute the optimal allocation policy as $P^{*}\left(g_{s s}, g_{s p}\right) \leq \min \left\{P_{p k}, \frac{Q_{p k}}{g_{s p}}\right\}$. Thus, the ergodic capacity in this case is maximized by transmitting at the following optimal power allocation policy:

$$
P^{*}\left(g_{s s}, g_{s p}\right)= \begin{cases}P_{p k}, & \text { if } g_{s p} \leq \frac{Q_{p k}}{P_{p k}}, \\ \frac{Q_{p k}}{g_{s p}}, & \text { otherwise. }\end{cases}
$$

From equation (10), we can see that, when the power gain $g_{s p}$ is lower than the ratio between both peak thresholds $\left(\frac{Q_{p k}}{P_{p k}}\right)$, the ST can transmit at its maximum power, $P_{p k}$, since this peak transmit power threshold satisfies the interference power constraint at PR. However, when $g_{s p}$ becomes larger than this ratio between both peak thresholds $\left(\frac{Q_{p k}}{P_{p k}}\right)$, the ST transmits with decreasing power values $\frac{Q_{p k}}{g_{s p}}$ which are inversely proportional to the power gain $g_{s p}$. Thus, we illustrate that it is beneficial to consider a severe fading channel between the ST and the PR in order to protect PR from SU interference and to maximize also the SU's capacity.

In Fig. 2, we represent the ergodic capacity under peak transmit and peak interference power constraints for a fixed $Q_{p k}=-5 \mathrm{~dB}$. We observe that when $P_{p k}=0$, the ergodic capacities for the four curves plotted in this figure are almost the same ${ }^{4}$. Obviously, this illustrate that the peak

\footnotetext{
${ }^{4}$ For Rayleigh fading channels, the power gains $g_{s s}$ and $g_{s p}$ are exponentially distributed with a unit mean $\sigma^{2}=1$. For AWGN channels, the power gains $g_{s s}$ and $g_{s p}$ follows a normal distribution law $\sim \mathcal{N}(0,1)$.
}

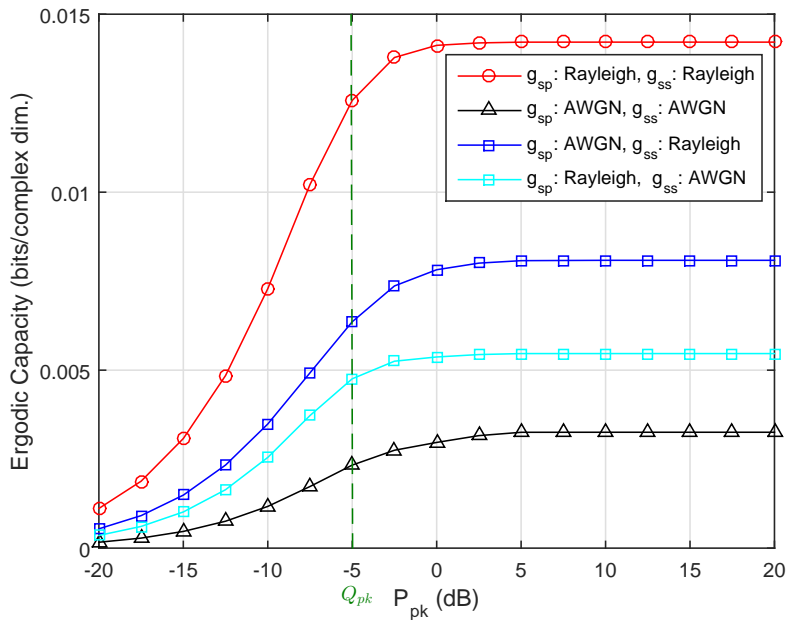

Fig. 2. The SU ergodic capacity vs the peak transmit power $P_{p k}$, where $Q_{p k}=-5 d B$ for various combination $\left(g_{s s}, g_{s p}\right)$ of AWGN and Rayleigh channels.

transmit power threshold $P_{p k}$ limits the performance of the CR network. However, when this peak transmit power threshold $P_{p k}$ is sufficiently high compared to the peak interference power threshold $Q_{p k}$, then the SU ergodic capacities vary. In this case, when the power gains $g_{s s}$ and $g_{s p}$ model the AWGN channels, the SU capacity is lower than when $g_{s s}$ models the AWGN channel and $g_{s p}$ models the Rayleigh fading channel. However, when the power gains $g_{s s}$ and $g_{s p}$ model the Rayleigh fading channels, the capacity for SU link is higher than when $g_{s s}$ models the Rayleigh fading channel and $g_{s p}$ models the AWGN channel. Thus, modelling with Rayleigh fading for the channel between the ST and the PR is an advantageous way in order to maximize the SU ergodic capacity.

Hereafter, we model both power gains $g_{s s}$ and $g_{s p}$ by the same channel model.

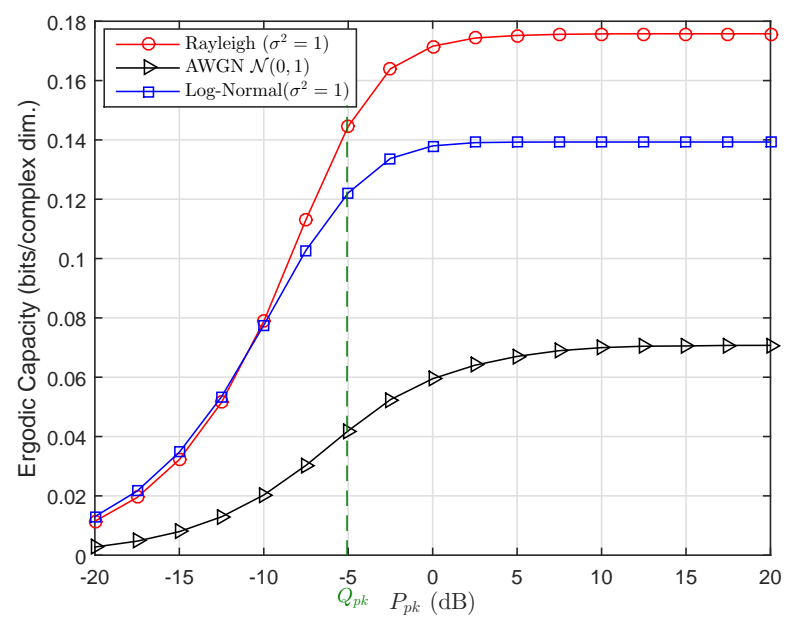

Fig. 3. The SU ergodic capacity vs the peak transmit power $P_{p k}$, where $Q_{p k}=-5 d B$ for Fading CR channels. 
In Fig. 3, we represent the ergodic capacity versus $P_{p k}$ under peak transmit and peak interference power constraints for different channel models ${ }^{45}$. We remark that the curve for Rayleigh fading channel outperforms the AWGN channel curve and outperforms also the Log-normal fading channel curve.

\section{B. Peak transmit and average interference power constraints}

In this case, the set of constraints in (9) becomes $\mathcal{F}_{2}$. The optimal power allocation is given by the following: $P^{*}\left(g_{s s}, g_{s p}\right)=$

$$
\begin{cases}0, & \text { if } \quad g_{s p} \geq \frac{K g_{s s}}{\mu N_{0}} \\ \frac{K}{\mu g_{s p}}-\frac{N_{0}}{g_{s s}} & \text { if } \quad \frac{K g_{s s}}{\mu N_{0}}>g_{s p}>\frac{K}{\mu\left(P_{p k}+\frac{N_{0}}{g_{s s}}\right)} \\ P_{p k}, & \text { if } \quad g_{s p} \leq \frac{K}{\mu\left(P_{p k}+\frac{N_{0}}{g_{s s}}\right)}\end{cases}
$$

where $K=\log _{2}(e)$ and $\mu$ is the non-negative Lagrange multiplier associated with the average interference power constraint (8) in $\mathcal{F}_{2}$. In order to compute $\mu$ at the optimum, there is two cases:

- If the average interference power constraint (8) in $\mathcal{F}_{2}$ is satisfied with strict inequality : $\mathbb{E}\left[g_{s p} P\left(g_{s s}, g_{s p}\right)\right]<Q_{a v}$, then $\mu$ must be equal to zero.

- Otherwise, $\mu$ can be obtained by substituting (11) into the constraint with equality: $\mathbb{E}\left[g_{s p} P\left(g_{s s}, g_{s p}\right)\right]=Q_{a v}$.

Obviously, from (11) we can remark that the optimal power depends on the power gains $g_{s s}$ and $g_{s p}$. In the middle interval, i.e., when $\frac{K g_{s s}}{\mu N_{0}}>g_{s p}>\frac{K}{\mu\left(P_{p k}+\frac{N_{0}}{g_{s s}}\right)}$, we can see that the optimal power allocation policy represents a water filling type of solution depending on both power gains $g_{s s}$ and $g_{s p}$.

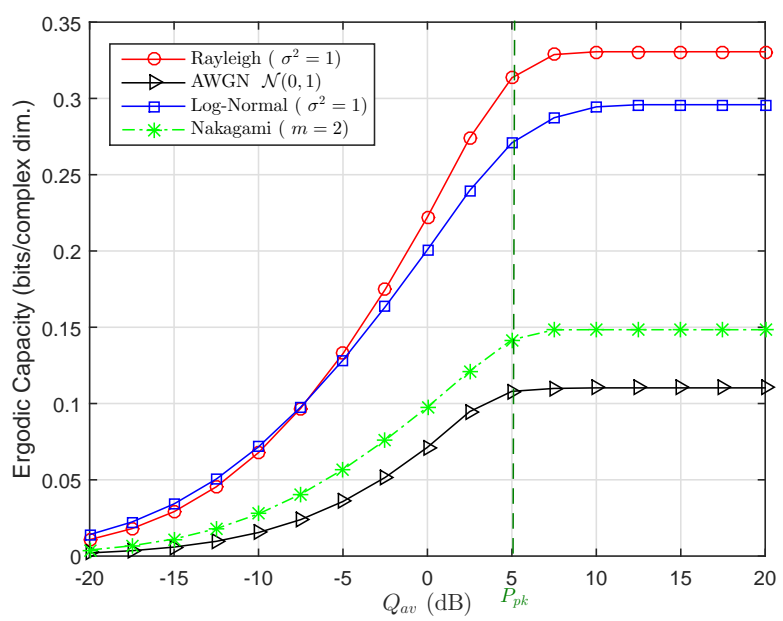

Fig. 4. The SU ergodic capacity vs the average interference power $Q_{a v}$ where $P_{p k}=5 d B$ for Fading CR channels.

In Fig. 4, we represent the ergodic capacity versus $Q_{a v}$ under peak transmit and average interference power constraints

${ }^{5}$ For Log-Normal fading channels, the power gains $g_{s s}$ and $g_{s p}$ are assumed to be null mean $\beta=0$ and unit variance $\sigma^{2}=1$. for different channel models ${ }^{456}$. We remark that the curve for Rayleigh fading channel outperforms all the others curves: for the AWGN channel, for the Log-normal fading channel and for the Nakagami fading channel. As we can see, the Log Normal fading curve is close to the Rayleigh fading curve when $Q_{a v}$ is small $\left(Q_{a v}<<P_{p k}\right)$.

\section{Average transmit and peak interference power constraints}

In this case, the set of constraints in (9) becomes $\mathcal{F}_{3}$. The optimal power allocation is given by the following: $P^{*}\left(g_{s s}, g_{s p}\right)=$

$$
\begin{cases}0, & \text { if } \quad g_{s s} \leq \frac{\lambda N_{0}}{K} \\ \frac{K}{\lambda}-\frac{N_{0}}{g_{s s}} & \text { if } \quad g_{s s}>\frac{\lambda N_{0}}{K}, g_{s p}<\frac{Q_{p k}}{\left(\frac{K}{\lambda}+\frac{N_{0}}{g_{s s}}\right)} \\ \frac{Q_{p k}}{g_{s p}}, & \text { if } \quad g_{s s}>\frac{\lambda N_{0}}{K}, g_{s p} \geq \frac{Q_{p k}}{\left(\frac{K}{\lambda}+\frac{N_{0}}{g_{s s}}\right)}\end{cases}
$$

where $K=\log _{2}(e)$ and $\lambda$ is the non-negative Lagrange multiplier associated with the average transmit power constraint (6) in $\mathcal{F}_{3}$. In order to compute $\lambda$ at the optimum, we obtain the following cases:

- If the average transmit power constraint (6) in $\mathcal{F}_{3}$ is satisfied with strict inequality: $\mathbb{E}\left[P\left(g_{s s}, g_{s p}\right)\right]<P_{a v}$, then $\lambda$ must be equal to zero.

- Otherwise, $\lambda$ can be obtained by substituting (12) into the average transmit power constraint with equality: $\mathbb{E}\left[P\left(g_{s s}, g_{s p}\right)\right]=P_{a v}$.

From (12) we can remark that, in the middle interval, i.e., when $g_{s s}>\frac{\lambda N_{0}}{K}, g_{s p}<\frac{Q_{p k}}{\left(\frac{K}{\lambda}+\frac{N_{0}}{g_{s}}\right)}$, the optimal power allocation policy represents a water filling type of solution depending only on the SU channel power gain $g_{s s}$.

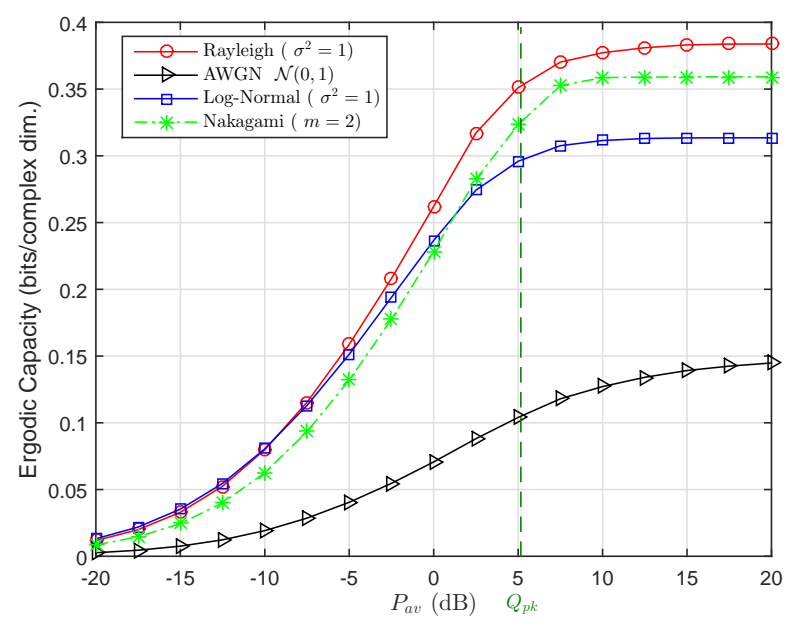

Fig. 5. The SU ergodic capacity vs the average transmit power $P_{a v}$ where $Q_{p k}=5 d B$ for Fading CR channels

In Fig. 5, we represent the ergodic capacity versus $P_{a v}$ under average transmit and peak interference power constraints for different channel models. We remark that the curve for

${ }^{6}$ For Nakagami fading channels, the channel power gains are assumed to be distributed with parameters $\omega=1, m=2$, and $\Gamma(2)=1$. 
Rayleigh fading channel outperforms the AWGN channel, the Log-normal fading channel and the Nakagami fading channel. As we can see, the Log Normal fading curve is close to the Rayleigh fading curve when $P_{a v}$ is small $\left(P_{a v}<<Q_{p k}\right)$. However, the Nakagami fading curve is close to the Rayleigh fading curve when $P_{a v}$ is high $\left(P_{a v}>>Q_{p k}\right)$.

For the previous problems, we remark that in practise the Rayleigh fading channels outperforms always the others types of fading channels: including Log-Normal fading and Nakagami fading models. Therefore, we will consider in the following problem only the Rayleigh fading channel model.

D. Average transmit and average interference power constraints

In this case, the set of constraints in (9) becomes $\mathcal{F}_{4}$. This case was studied in details in [19]. Using the Lagrangian method [20] and since the problem is strictly concave, by applying the Karush Kuhn Tucker (KKT) conditions, we get the optimal power: ${ }^{7}$

$$
P^{*}\left(g_{s s}, g_{s p}\right)=\left(\frac{K}{\lambda+\mu g_{s p}}-\frac{N_{0}}{g_{s s}}\right)^{+},
$$

where $\lambda$ and $\mu$ represent the non negative Lagrangian multipliers associated to the average transmit power constraint and the average interference power constraint, respectively. Since it is difficult to solve analytically the non-negative Lagrange multipliers $\lambda$ and $\mu$ where both average transmit power and average interference power constraints must be satisfied with equalities, then we use a decoupling method where we decouple the original problem (9) subject to the set constraint $\mathcal{F}_{4}$ into two sub-problems, which are easily solved individually [19].

- Sub-Problem 1:

$$
\left(S P_{1}\right) \begin{cases}\max _{P\left(g_{s s}, g_{s p}\right) \geq 0} & \mathbb{E}\left[\log _{2}\left(1+\frac{g_{s s} P\left(g_{s s}, g_{s p}\right)}{N_{0}}\right)\right] \\ \text { s.t. } & \mathbb{E}\left[P\left(g_{s s}, g_{s p}\right)\right] \leq P_{a v} .\end{cases}
$$

This problem is equivalent to the problem in section VI.C with no peak interference power constraint i.e., $Q_{p k} \rightarrow$ $+\infty$. Similar to (12), the optimal power solution for $\left(S P_{1}\right)$, denoted by $P^{(1)}\left(g_{s s}, g_{s p}\right)$, is given by:

$$
P^{(1)}\left(g_{s s}, g_{s p}\right)=\left(\frac{K}{\lambda}-\frac{N_{0}}{g_{s s}}\right)^{+},
$$

where $\lambda$ satisfy the average transmit power constraint with equality : $\mathbb{E}\left[P^{(1)}\left(g_{s s}, g_{s p}\right)\right]=P_{a v}$. Therefore, we obtain the non-negative Lagrange multiplier $\lambda$ as follows:

$$
\lambda=\frac{K}{P_{a v}+\frac{N_{0}}{g_{s s}}} .
$$

- Sub-Problem 2:

$$
\left(S P_{2}\right) \begin{cases}\max _{P\left(g_{s s}, g_{s p}\right) \geq 0} & \mathbb{E}\left[\log _{2}\left(1+\frac{g_{s s} P\left(g_{s s}, g_{s p}\right)}{N_{0}}\right)\right] \\ \text { s.t. } & \mathbb{E}\left[g_{s p} P\left(g_{s s}, g_{s p}\right)\right] \leq Q_{a v} .\end{cases}
$$

This problem is equivalent to the problem in section VI.B with no peak transmit power constraint i.e., $P_{p k} \rightarrow+\infty$.

\footnotetext{
${ }^{7}$ We denote by $(x)^{+}=\max (0, x)$.
}

Similar to (11), the optimal power solution for $\left(S P_{2}\right)$, denoted by $P^{(2)}\left(g_{s s}, g_{s p}\right)$, is given by:

$$
P^{(2)}\left(g_{s s}, g_{s p}\right)=\left(\frac{K}{\mu g_{s p}}-\frac{N_{0}}{g_{s s}}\right)^{+},
$$

where $\lambda$ satisfy the average interference power constraint with equality : $\mathbb{E}\left[g_{s p} P^{(2)}\left(g_{s s}, g_{s p}\right)\right]=Q_{a v}$. Therefore, we obtain the non-negative Lagrange multiplier $\mu$ as follows:

$$
\mu=\frac{K}{Q_{a v}+\frac{N_{0} g_{s p}}{g_{s s}}} .
$$

Therefore, we solve the initial problem and calculate $P^{*}\left(g_{s s}, g_{s p}\right)$ from the two optimal allocations.

Remark IV.1. 1. We remark that the constraints $\mathbb{E}\left[P^{(2)}\left(g_{s s}, g_{s p}\right)\right] \leq P_{a v}$ and $\mathbb{E}\left[g_{s p} P^{(1)}\left(g_{s s}, g_{s p}\right)\right] \leq Q_{a v}$ can not be simultaneously satisfied.

2. If the optimal power allocation for $\left(S P_{1}\right)$ which satisfy the average transmit power, satisfy also the average interference power constraint : $\mathbb{E}\left[g_{s p} P^{(1)}\left(g_{s s}, g_{s p}\right)\right] \leq$ $Q_{a v}$, then the optimal power allocation for the initial problem is the optimal solution for $\left(S P_{1}\right): P^{*}\left(g_{s s}, g_{s p}\right)=$ $P^{(1)}\left(g_{s s}, g_{s p}\right)$

3. If the optimal power allocation for $\left(S P_{2}\right)$ which satisfy the average interference power constraint, satisfy also the average transmit power constraint : $\mathbb{E}\left[P^{(2)}\left(g_{s s}, g_{s p}\right)\right] \leq$ $P_{a v}$, then the optimal power allocation for the initial problem is the optimal solution for $\left(S P_{2}\right): P^{*}\left(g_{s s}, g_{s p}\right)=$ $P^{(2)}\left(g_{s s}, g_{s p}\right)$

4. If remark 2. and 3. are not satisfied then, we use the Lagrangian method [20] and we give an iterative algorithm based on the sub-gradient method [17]. Thus, we find $\lambda$ and $\mu$ that satisfy: $\mathbb{E}\left[P^{*}\left(g_{s s}, g_{s p}\right)\right]=P_{a v}$ and $\mathbb{E}\left[g_{s p} P^{*}\left(g_{s s}, g_{s p}\right)\right]=Q_{\text {av }}$ respectively.

Based on these remarks, we can solve the initial problem using the following algorithm:

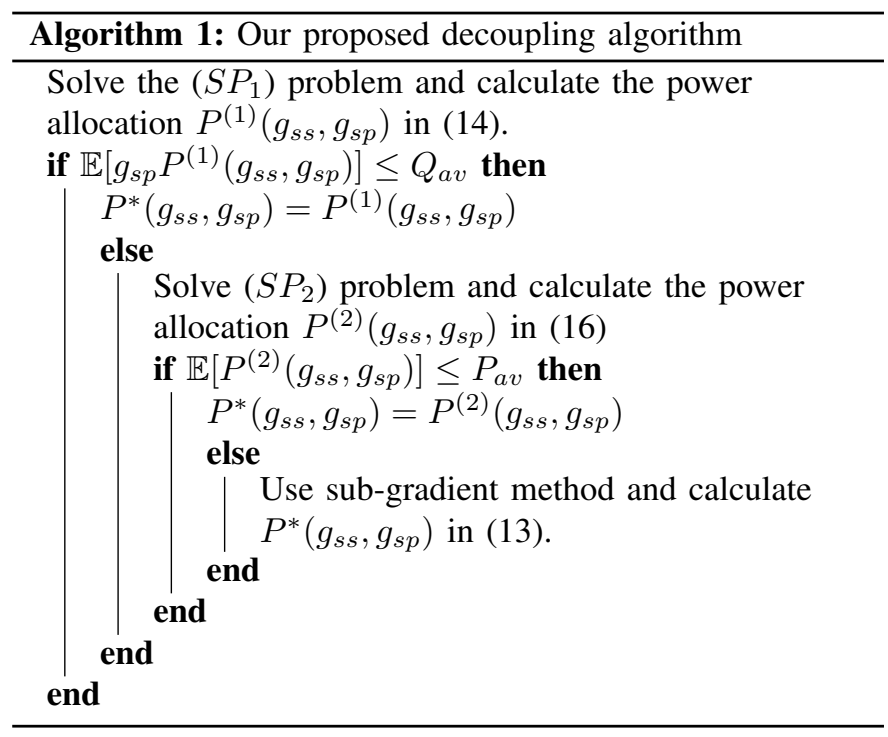

This decoupling method reduces the complexity of the initial 
problem by replacing the study of this general problem to two equivalent decoupled problems which are more easier to solve. In practice, we remark that, in most cases $P^{(1)}\left(g_{s s}, g_{s p}\right)$ in (14) or $P^{(2)}\left(g_{s s}, g_{s p}\right)$ in (16) is the optimal solution for our initial problem.

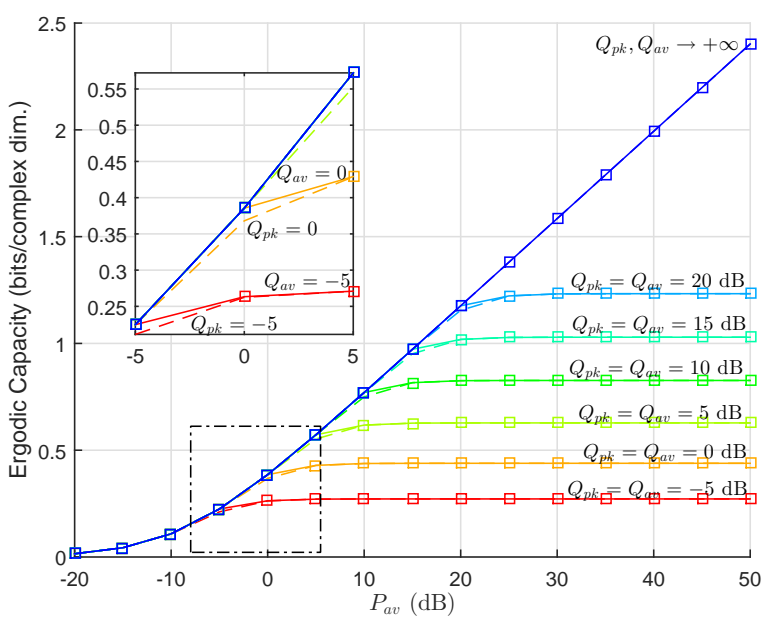

Fig. 6. The maximum achievable capacity of the SU vs average power $P_{a v}$ where $Q_{p k}$ and $Q_{a v} \in\{-5,0,5,10,15,20,+\infty\} d B$ for a Rayleigh Fading channel.

In Fig. 6, we compare the maximum achievable capacity of the SU in problem VI.D with the one in problem VI.C. We plot also the curve with no average interference power constraint $Q_{a v}=+\infty$ and the curve with no peak interference power constraint $Q_{p k}=+\infty$.

In one hand, we can see that when $P_{a v}$ is small $\left(P_{a v}<<Q_{a v}\right)$, the capacities for $Q_{a v} \in\{-5,0,5,10,15,20,+\infty\} d B$ do not vary much. Thus, $Q_{a v}$ limits the SU achievable capacity. However, when $P_{a v}$ is sufficiently high compared to $Q_{a v}$ (i.e., $P_{a v}>>Q_{a v}$ ), the capacities become flat. This illustrate that, in this case, $P_{a v}$ becomes the dominant constraint.

On the other hand, we plot in the same figure the ergodic capacity under average transmit power and peak interference power (defined in problem VI.C). We remark that the ergodic capacity with average interference constraint outperforms the one with peak interference constraint when $P_{a v}$ is nearby $Q_{a v}$.

\section{CONCLUSION}

In this paper, a cognitive radio system is considered. In the spectrum sharing context, the optimal power allocation strategies to achieve the SU ergodic capacity under different types of power constraints and fading channel models are studied. We have shown that modelling with Rayleigh fading for the ST-PR channel is an advantageous way to ameliorate the SU ergodic capacity. Furthermore, we have considered four combinations of power constraints, since the interference power constraint and the transmit power constraint can be restricted by a peak or an average threshold. We have also shown that the SU ergodic capacity under average transmit power constraint and average interference power constraint outperforms the one with peak power constraints. In this case, we have proposed a decoupling method. Our method have reduced the complexity of the initial problem and made our initial problem easier to solve. Future works can include the case where several SUs coexist in the primary networks. We will provide in the future a joint scheduling spectrum and power allocation problem similar to [17].

\section{REFERENCES}

[1] "Spectrum Policy Taskforce Report", Technical report, Fed. Comm. Commission, Nov. 2002.

[2] J. Mitola and G. Q. Maguire, "Cognitive radio: making software radios more personal”, IEEE Pers. Commun., vol. 6, no. 6, pp. 13-18, Aug. 1999.

[3] S. Haykin, "Cognitive radio: brain-empowered wireless communications", IEEE J. Select. Areas Commun., vol. 23, no. 2, pp. 201-220, Feb. 2005.

[4] Y.-C. Liang, Y. Zeng, E. C. Y. Peh, and A. T. Hoang, "Sensingthroughput tradeoff for cognitive radio networks", IEEE Trans. Wireless Commun., vol. 7, no. 4, pp. 1326-1337, Apr. 2008.

[5] J.M. Peha, "Approaches to Spectrum Sharing", IEEE Comm. Magazine, vol. 43, no. 2, pp. 10-12, Feb. 2005.

[6] A. Ghasemi and E. S. Sousa, "Fundamental limits of spectrum-sharing in fading environments", IEEE Trans. Wireless Commun., vol.6, no.2, pp. 649-658, Feb. 2007.

[7] D. Cabric and R. W. Brodersen. "Cognitive radio spectrum sharing technology", Wireless Technologies, Circuits, Systems, and Devices, pp. 131-157, 2017.

[8] M. Hawa, A. AlAmmouri, A. Alhiary and N. Alhamad, "Distributed opportunistic spectrum sharing in cognitive radio networks", International journal of communication systems, vol. 30, no.7, 2017.

[9] L. Musavian and S. Aissa, "Ergodic and outage capacities of spectrum sharing systems in fading channels", IEEE Global Telecommunications Conference (GLOBECOM), Washington. DC, USA, pp. 3327-3331, 2007.

[10] Zhang, "On peak versus average interference power constraints for protecting primary users in cognitive radio networks", IEEE Trans. Wireless Commun., vol. 8, no. 4, pp. 11281138, Apr. 2009.

[11] X. Kang, R. Zhang, Y. C. Liang, and H. K. Garg, "Optimal power allocation strategies for fading cognitive radio channels with primary user outage constraint”, IEEE J. Sel. Areas Commun., vol. 29, no. 2, pp. 374383, Feb. 2011.

[12] F. Zhou, N. C. Beaulieu,Z. Li, J. Si and P. Qi, "Energy-efficient optimal power allocation for fading cognitive radio channels: Ergodic capacity, outage capacity, and minimum-rate capacity", IEEE Transactions on Wireless Communications, vol. 15, no. 4, pp. 2741-2755. 2016.

[13] L. Musavian and S. Aissa, "Capacity and power allocation for spectrum sharing communications in fading channels", IEEE Trans. Wireless Commun., vol. 8, no. 1, pp. 148156, Jan. 2009.

[14] X. Kang, Y. C. Liang, A. Nallanathan, H. K. Garg, and R. Zhang, "Optimal power allocation for fading channels in cognitive radio networks: Ergodic capacity and outage capacity", IEEE Transactions on Wireless Communications, 8(2), pp. 940-950, 2009.

[15] R. Masmoudi, E. V. Belmega, I. Fijalkow, and N. Sellami, "A closedform solution to the power minimization problem over two orthogonal frequency bands under QoS and cognitive radio interference constraints", IEEE DySPAN, pp. 212-222, USA, Oct. 2012.

[16] R. Masmoudi, E.V. Belmega , I. Fijalkow and N. Sellami, "A unifying view on energy-efficiency metrics in cognitive radio channels", European Signal Processing Conference, pp. 171-175, Sep. 2014.

[17] R. Masmoudi, E. V. Belmega and I. Fijalkow, "Efficient spectrum scheduling and power management for opportunistic users", EURASIP Journal on Wireless Communications and Networking (JWCN), vol. 2016, no. 97, pp. 1-19, Apr. 2016.

[18] L. Musavian, and T. LeNgoc, "QoS-based power allocation for cognitive radios with AMC and ARQ in Nakagami-m fading channels", Transactions on Emerging Telecommunications Technologies, vol. 27, no. 2, pp. 266-277, 2016.

[19] R. Masmoudi, "Power allocation Problem for Fading Channels in Cognitive Radio Networks", International Multi-Conference on Systems, Signals and Devices (SSD), Mar. 2018.

[20] S.Boyd and L. Vandenberghe, "Convex Optimization", Cambridge Univ Press, Cambridge, UK, 2004. 